\title{
Versican: a novel modulator of hepatic fibrosis
}

\author{
Terence N Bukong ${ }^{1}$, Sean B Maurice ${ }^{1,2}$, Barinder Chahal ${ }^{1,3}$, David F Schaeffer ${ }^{4}$ and Paul J Winwood ${ }^{1,3}$
}

Little is known about the deposition and turnover of proteoglycans in liver fibrosis, despite their abundance in the extracellular matrix. Versican plays diverse roles in modulating cell behavior in other fibroproliferative diseases, but remains poorly described in the liver. Hepatic fibrosis was induced by carbon tetrachloride treatment of C57BL/6 mice over 4 weeks followed by recovery over a 28-day period. Primary mouse hepatic stellate cells (HSCs) were activated in culture and versican was transiently knocked down in human (LX2) and mouse HSCs. Expression of versican, A Disintegrinlike and Metalloproteinase with Thrombospondin-1 motifs (ADAMTS)-1, -4, -5, -8, -9, -15, and -20, and markers of fibrogenesis were studied using immunohistochemistry, real-time quantitative $P C R$, and western blotting. Immunohistochemistry showed increased expression of versican in cirrhotic human livers and the mouse model of fibrosis. Carbon tetrachloride treatment led to significant increases in versican expression and the proteoglycanases ADAMTS-5, $-9,-15$, and -20, alongside TNF- $\alpha$, $a-$-smooth muscle actin (a-SMA), collagen- 1 , and TGF- $\beta$ expression. During recovery, expression of many of these genes returned to control levels. However, expression of ADAMTS-5, $-8,-9$, and -15 showed delayed increases in expression at 28 days of recovery, which corresponded with decreases in versican V0 and V1 cleavage products (G1-DPEAAE ${ }^{1401}$ and G1-DPEAAE ${ }^{441}$ ). Activation of primary HSCs in vitro significantly increased versican, a-SMA, and collagen-1 expression. Transient knockdown of versican in HSCs led to decreases in markers of fibrogenesis and reduced cell proliferation, without inducing apoptosis. Versican expression increases during HSC activation and liver fibrosis, and proteolytic processing occurs during the resolution of fibrosis. Knockdown studies in vitro suggest a possible role of versican in modulating hepatic fibrogenesis.

Laboratory Investigation (2016) 96, 361-374; doi:10.1038/labinvest.2015.152; published online 11 January 2016

Chronic liver disease remains a major public health problem worldwide $^{1-5}$ and is complicated by progressive fibrosis and cirrhosis. Chronic liver injury, irrespective of etiology, is typically characterized by inflammation and increased deposition of extracellular matrix (ECM) with a greater proportion of fibrillar collagens. This fibrotic ECM leads to adverse functional alterations in the liver, including portal hypertension and liver failure secondary to hepatocellular dysfunction. ${ }^{6-10}$ The accumulation of fibrotic tissue results mainly from sustained activation of hepatic stellate cells (HSCs) to a fibroblastic phenotype, in response to many factors, including stimulation by proinflammatory and fibrogenic cytokines (particularly TGF- $\beta$ ), tissue hypoxia, reactive oxygen species, innate immune responses via interaction with Toll-like receptors (TLRs), the stiffness of the ECM, and ECM/fibroblast interactions. ${ }^{5,11-17}$ During the fibroproliferative stage, treatment of the underlying cause of liver disease results in the best prognostic outcome with disease reversal occurring in many patients. ${ }^{18-21}$ Matrix metalloproteinase (MMP)-mediated degradation of fibrillar collagens is a well-established aspect of recovery from liver fibrosis in both experimental models and human disease, with a majority of the MMPs being synthesized by HSCs. ${ }^{7,22-24}$ The tissue inhibitor of metalloproteinase (TIMP) family of endogenous inhibitors is involved in regulating the activity of MMPs and collectively, MMP and TIMP activity contribute to regulation of HSC activity during fibrogenesis and recovery. ${ }^{25-29}$ While the changes in collagen type I and III turnover have been well described in liver fibrosis, less is known about changes in proteoglycans that are the most abundant component of the ECM.

Proteoglycans are a diverse group of highly glycosylated proteins composed of a core protein covalently linked to a variable number of negatively charged, sulfated glycosaminoglycan side chains. Proteoglycans have numerous biological functions in the ECM including modulating

\footnotetext{
${ }^{1}$ Northern Medical Program, University of Northern British Columbia, Dr. Donald Rix Northern Health Sciences Centre, Prince George, BC, Canada; ${ }^{2}$ Department of Cellular and Physiological Sciences, Faculty of Medicine, University of British Columbia, BC, Canada; ${ }^{3}$ Faculty of Medicine, University of British Columbia, BC, Canada and ${ }^{4}$ Department of Pathology and Laboratory Medicine, Faculty of Medicine, University of British Columbia, BC, Canada

Correspondence: Dr PJ Winwood, BSc, MBBS, DM, Northern Medical Program, University of Northern British Columbia, Dr. Donald Rix Northern Health Sciences Centre, 3333 University Way, Prince George, BC V2N 4Z9, Canada.

E-mail: Paul.Winwood@unbc.ca

Received 15 July 2015; revised 29 October 2015; accepted 2 November 2015
} 
cell/tissue homeostasis, cell adhesion/migration, as well as regulation of cellular signaling. ${ }^{30}$ Early work has established that heparan sulfate proteoglycans are more abundant than chondroitin sulfate proteoglycans (CSPGs) in the normal liver and play important roles in maintaining hepatocyte phenotype. ${ }^{31-33}$ However, under remodeling conditions, CSPGs are increased much more significantly, which could reflect a modulatory role. ${ }^{31,32}$ The small leucine-rich proteoglycans decorin, biglycan, and lumican have all been associated with hepatic fibrosis. ${ }^{34,35}$ Early studies identified the large CSPG versican in the liver and suggested that expression increases with fibrosis. ${ }^{36-38}$ Versican is overexpressed in patients with non-alcoholic steatohepatitis, compared with obese control patients ${ }^{39}$ and we have recently described versican upregulation in HSCs activated in culture. ${ }^{40}$ Much remains to be elucidated about the roles of specific CSPGs in the liver.

Versican is a large, alternatively spliced, aggregating CSPG with a molecular weight that can be $>1000 \mathrm{kDa}$ and a ubiquitous pattern of expression. ${ }^{36,41}$ It is essential in development, wherein versican null mice die during embryogenesis. ${ }^{42}$ Conditional knockout of versican has demonstrated its importance both during chondrogenesis and in binding the profibrotic cytokine TGF- $\beta .{ }^{43}$ Versican is a major component of the early fibroproliferative matrix in lung fibrosis where its deposition is associated with fibroblast migration and proliferation, preceding collagen deposition. ${ }^{44-46}$ It is also associated with the neointima in atherosclerosis and restenosis. ${ }^{47}$ Versican is present in the stroma of many different forms of cancer, including liver cancer, where its expression appears to correlate reliably with growth, invasion, and metastatic potential. ${ }^{48-50}$ Versican has been shown to play modulatory roles in cell proliferation, differentiation, adhesion, and the homeostatic integrity of the ECM. ${ }^{48,51}$ It is a central component of inflammatory processes and is involved in multiple protein-binding interactions and modulation of leukocytes and lympohocytes. ${ }^{52,53}$ Versican has also been shown to activate macrophages through toll-like receptor 2 (TLR2) and its co-receptors, TLR6 and CD14, leading to the production of the proinflammatory cytokine TNF- $\alpha$, in addition to macrophage activation. ${ }^{54}$

Proteolytic processing of versican occurs by several different members of the ADAMTS (A Disintegrin-like and Metalloproteinase with Thrombospondin-1 motifs) family of enzymes. ADAMTS- $1,-4,-5,-9,-15$, and -20 have all been demonstrated to cleave versican ${ }^{55-58}$ and by homology, ADAMTS- 8 is presumed to also. This cluster of ADAMTS enzymes represents slightly less than half of the ADAMTS family and is considered the proteoglycan cleaving 'super-clade'. ${ }^{59}$ ADAMTS processing of versican produces several cleavage sites, ${ }^{55,60,61}$ the best known of these is the DPEAAE $\downarrow$ ARRGQ site. $^{55}$ Cleavage at this site releases the $\mathrm{N}$-terminal globular domain of versican (the G1 domain) and a portion of the GAG- $\beta$ domain, leading to a 441 amino acid
$\mathrm{N}$-terminal fragment of the V1 isoform (human or mouse) with several potential glycosylation sites. ${ }^{41,62}$ In the larger versican V0 isoform, the GAG- $\alpha$ domain is also included in this cleaved portion leading to a 1428 residue $\mathrm{N}$-terminal fragment in human versican or 1401 in mouse, that is predicted to contain 5-8 glycosaminoglycan side chains, in addition to several $\mathrm{N}$ - and O-linked glycosylations. ${ }^{36,62}$ Neo-epitope antibodies to the C-terminal DPEAAE sequence have permitted investigation of this G1-DPEAAE cleavage product in tissue remodeling processes. Proteolytic processing of versican is essential in a number of developmental processes and generates bioactive fragments including G1-DPEAAE ${ }^{441} \cdot{ }^{63-68}$ It is becoming increasingly clear that the regulated expression and proteolytic processing of versican are critically involved in influencing cell phenotype during inflammation and remodeling. ${ }^{51,69}$ Bioactive ECM proteolytic fragments are increasingly recognized to regulate cellular function and have been generally referred to as matrikines. $^{70}$ Recently, the G1-DPEAAE ${ }^{441}$ neo-epitope cleavage product of versican V1 has been dubbed 'versikine' to underscore the importance of versican cleavage in general and this versican cleavage product, in particular, in regulating tissue remodeling. ${ }^{71}$ Given the known roles of versican in other tissues and the increases in CSPGs in liver remodeling, we hypothesized that versican expression would also play a role in the pathogenesis of hepatic fibrosis.

Here, we report that versican is dysregulated during liver fibrosis and reduces during recovery from liver injury. Furthermore, gene interference studies suggest that a decrease in versican expression is associated with deactivation of HSCs and a concomitant reduction of collagen expression, which would lead to a reduction of synthesis and deposition of fibrotic ECM.

\section{MATERIALS AND METHODS Human Tissue Sample Collection}

Human tissues were obtained through the BC Gastrointestinal Biobank from surplus materials obtained at the time of hepatectomy. Clinical samples of non-cirrhotic liver were from patients with metastatic colorectal carcinoma $(n=4)$ or metastatic breast carcinoma $(n=1)$, from patients aged 31 to 83. Clinical samples of cirrhotic liver were from patients with autoimmune hepatitis $(n=2)$, steatohepatitis $(n=1)$, primary sclerosing cholangitis $(n=1)$, and hepatocellular carcinoma $(n=1)$, from patients aged 27 to 63 .

\section{Induction of Liver Fibrosis in Mice with Carbon Tetrachloride Treatment}

Three-week-old male and female C57BL/6 mice purchased from Charles River Laboratories (Senneville, QC, Canada) were housed in a temperature-humidity-controlled environment with $12 \mathrm{~h}$ light-dark cycles and had unrestricted access to food and water. Liver fibrosis was induced by intraperitoneal injection (i.p.) of $0.9 \mathrm{ml} / \mathrm{kg}$ carbon tetrachloride $\left(\mathrm{CCl}_{4}\right)$ dissolved in olive oil (1:1) twice weekly 
Table 1 qPCR primer sequences

\begin{tabular}{|c|c|c|c|c|c|}
\hline Target gene & Species & Accession & Forward primer $\left(5^{\prime}-3^{\prime}\right)$ & Reverse primer $\left(5^{\prime}-3^{\prime}\right)$ & Product (bp) \\
\hline Adamts 1 & Mus musculus & NM_009621 & CATAACAATGCTGCTATGTGCG & TGTCCGGCTGCAACTTCAG & 167 \\
\hline Adamts5 & Mus musculus & NM_011782 & GGAGCGAGGCCATTTACAAC & CGTAGACAAGGTAGCCCACTTा & 110 \\
\hline Adamts8 & Mus musculus & NM_013906 & GGCAACGGCGTITCAACAAG & CTGAGAACGTGCCCTAGCTC & 223 \\
\hline Adamts 20 & Mus musculus & NM_177431 & GTCCTGGGAAGTTCGTTTCCA & GGCTGAAATGCCGGTTCTG & 128 \\
\hline Vcan & Mus musculus & NM_001081249 & TITTACCCGAGTTACCAGACTCA & GGAGTAGTTGTTACATCCGTTGC & 106 \\
\hline Collar & Mus musculus & NM_007742 & GCTCCTCTTAGGGGCCACT & CCACGTCTCACCATTGGGG & 103 \\
\hline Acta2 & Mus musculus & NM_007392 & GTCCCAGACATCAGGGAGTAA & TCGGATACTTCAGCGTCAGGA & 102 \\
\hline Tnf & Mus musculus & NM_013693 & CATCTTCTCAAAATTCGAGTGACAA & TGGGAGTAGACAAGGTACAACCC & 175 \\
\hline Gapdhs & Mus musculus & NM_001290631 & TGGATTTGGACGCATTGGTC & TITGCACTGGTACGTGTTGAT & 211 \\
\hline Actb & Mus musculus & NM_007393 & GGCTGTATTCCCCTCCATCG & CCAGTTGGTAACAATGCCATGT & 154 \\
\hline Rn18s & Mus musculus & NR_003278 & GTAACCCGTTGAACCCCATT & CCATCCAATCGGTAGTAGCG & 151 \\
\hline RNA18S5 & Homo sapiens & NR_003286 & GTAACCCGTTGAACCCCATT & CCATCCAATCGGTAGTAGCG & 151 \\
\hline VCAN & Homo sapiens & NM_004385 & GTAACCCATGCGCTACATAAAGT & GGCAAAGTAGGCATCGTTGAAA & 110 \\
\hline NIFK & Homo sapiens & NM_032390 & TTCGCAAGCGCATAACCCA & AACCGTGTCACAGTGCCAAA & 136 \\
\hline COLIA1 & Homo sapiens & NM_000088 & GAGGGCCAAGACGAAGACATC & CAGATCACGTCATCGCACAAC & 140 \\
\hline ACTA2 & Homo sapiens & NM_001141945 & GTGTTGCCCCTGAAGAGCAT & GCTGGGACATTGAAAGTCTCA & 109 \\
\hline
\end{tabular}

Abbreviations: Acta2/ACTA2, a-smooth muscle actin; Actb, actin beta; Rn18s, $18 \mathrm{~S}$ ribosomal RNA; ADAMTS, a disintegrin-like and metalloproteinase with thrombospondin-1 motifs; Col1a1/COL1A1, collagen type I, alpha 1; Gapdhs, glyceraldehyde-3-phosphate dehydrogenase, spermatogenic; NIFK, nucleolar protein interacting with the FHA domain of MKI67; Tgfb1/TGFB1, transforming growth factor beta 1; Timp, tissue inhibitor of metalloproteinase; Tnf, tumor necrosis factor; RNA18S5, RNA 18 S ribosomal 5; Vcan/VCAN, versican.

for 4 weeks. Mice treated with i.p. olive oil vehicle over 8 weeks served as controls. Following $\mathrm{CCl}_{4}$ treatment mice were killed or allowed to recover over 3, 7, or 28 days and then killed to assess the extent of liver fibrosis and recovery. ${ }^{26}$ There were 10 mice in each treatment and the control group although some attrition meant that statistical analysis was undertaken on the results from 9 to 10 animals per group. Maximum fibrosis was considered to be 3 days after last instillation of $\mathrm{CCl}_{4}$ as previously described, ${ }^{26}$ and recovery times were measured from that point. A portion of excised liver was fixed in $10 \%$ phosphate-buffered formalin for histological analysis. The remaining portions of the liver were collected in RNase-free tubes and snap-frozen in liquid nitrogen for further analysis as subsequently described.

\section{Ethics Statement}

Human tissues were obtained through the BC Gastrointestinal Biobank at Vancouver General Hospital (UBC REB \#
H12-03476). Patients gave consent to research use with surplus materials prior to hepatectomies being performed. Clinical data were anonymized for further research purposes. Animal work performed in this study was approved by the Animal Care and Use Committee of the University Northern British Columbia and conformed to institutional guidelines for animal experimentation (protocol: A2010.0318.006). Terminal surgeries were performed under deep anesthesia with isoflurane and all efforts were made to minimize suffering.

\section{Histological Assessment}

Immunohistochemical staining for versican was performed in all cases included in this study on 4 - $\mu$ m-thick paraffin sections of whole-tissue sections on the semi-automated Ventana Discovery XT instrument (Ventana Medical Systems, Tucson, AZ, USA). Antigen retrieval was standard CC1: versican rabbit polyclonal (clone HPA004726; Sigma, 
a
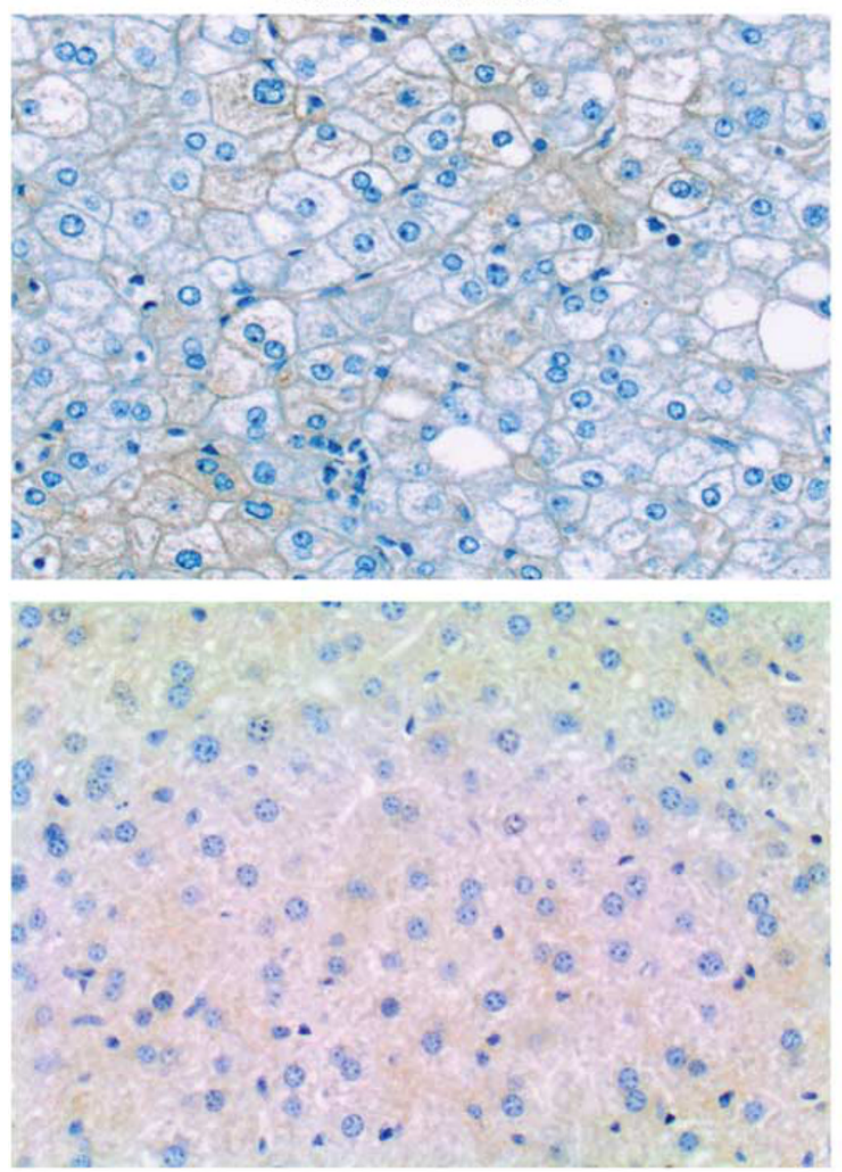

Mouse Control
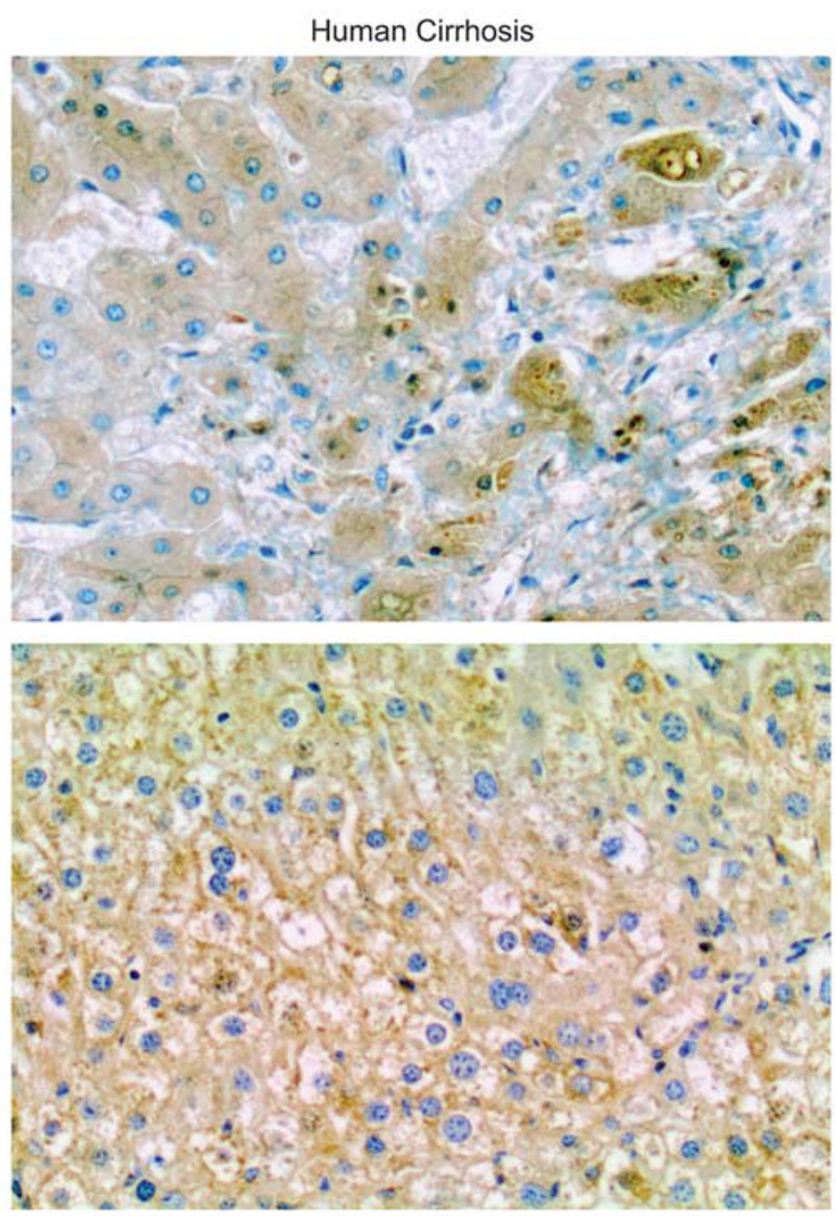

Mouse Fibrosis

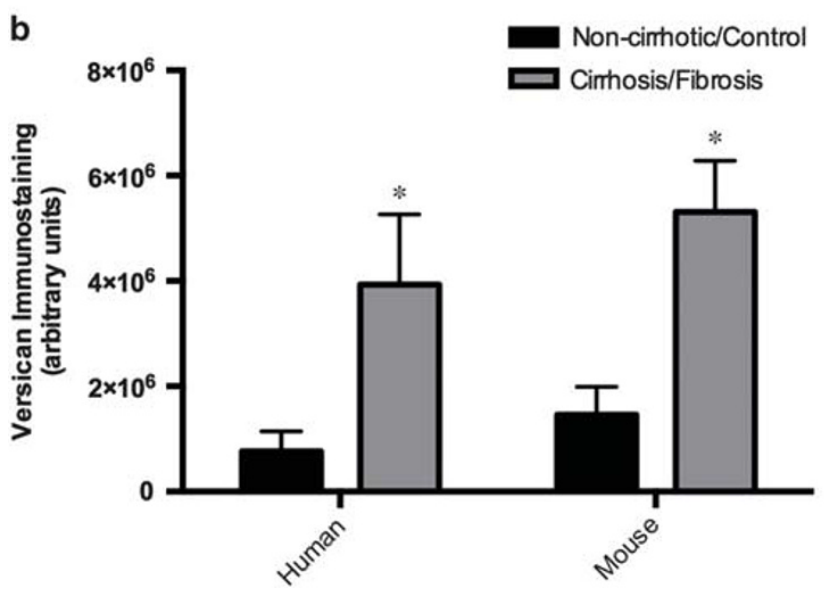

Figure 1 Versican expression in human liver cirrhosis and mouse liver fibrosis. (a) Sections of liver parenchyma from non-cirrhotic $(n=5)$ and cirrhotic $(n=5)$ human livers were stained using immunohistochemistry for versican (12C5), alongside normal $(n=5)$ and fibrotic $(n=5)$ mouse liver sections (after 4 weeks $\mathrm{CCl}_{4}$ ) (HPA004726). Representative non-cirrhotic human liver shown from a patient with metastatic colorectal carcinoma and representative cirrhotic human liver shown from a patient with primary sclerosing cholangitis. (b) Quantitation of versican immunostaining was performed with image thresholding and significance determined by two-way analysis of variance $\left(^{*}\right)(P<0.05)$. Mean value per lane is shown $(n=5)$ and error bars represent the s.e.m. $\mathrm{CCL}_{4}$, carbon tetrachloride.

St Louis, MO, USA) or versican mouse monoclonal (clone 12C5; Developmental Studies Hybridoma Bank, University of Iowa $)^{72}$ was applied at 1:100, followed by incubation with prediluted UltraMap anti-mouse or anti-rabbit HRP secondary, and detection with Ventana UltraMap DAB kit (Ventana). Quantitation was performed on each image with the investigator blinded to group assignment $(n=5$ samples per condition). Pathological fibrosis staging was performed 
on sections stained with Masson's trichrome as per the established protocol and analyzed by a gastrointestinal pathologist (DFS), blinded to clinical parameters, according to Ishak-Knodell staging. Collagen quantitation was performed on picrosirius red stained sections with 10 random fields for each slide ( $n=9-10$ animals for each time point), measured in arbitrary units. Slides were imaged using a bright-field Olympus BX61 microscope. Image thresholding was performed with cellSens Dimensions 1.6 software (Olympus, PA) with parameters determined empirically and used identically across all slides.

\section{Primary Stellate Cell Isolation and Activation}

Primary mouse HSCs were isolated as previously described $^{73-76}$ with slight modification. Briefly, isotonic Hanks' Balanced Salt Solution was used to perfuse livers of anesthetized C57BL/6 mice. Following liver perfusion, mouse livers were excised and finely chopped in DNAse1. Chopped liver samples were then treated with pronase (Roche cat. \# 10165921001) and collagenase (Roche cat. \# 11088815001) solution in a $50-\mathrm{ml}$ sterile tube and incubated with gentle shaking at $37^{\circ} \mathrm{C}$ in a water bath for $20 \mathrm{~min}$. Following enzyme treatment, liver digests were filtered through a cell strainer and washed with Hanks supplemented with DNAse1. Primary HSCs were then separated by density gradient centrifugation with OptiPrep (Sigma). Primary culture mouse HSCs were cultured on plastic culture flasks in the presence of $10 \%$ fetal calf serum over the subsequent indicated time periods.

\section{RNA Extraction and Real-Time Quantitative PCR}

Total RNA from cells or tissue samples was extracted using the RNeasy kit (Qiagen) according to the manufacturer's protocol. Quantitation and estimation of RNA purity was carried out using the NanoDrop (Thermo Scientific) spectrophotometer readings at 260 and $280 \mathrm{~nm}$. Reverse transcription of $1 \mu \mathrm{g}$ RNA was conducted for $30 \mathrm{~min}$ at $42{ }^{\circ} \mathrm{C}$ using iScript (Bio-Rad). Quantification of target gene mRNAs was undertaken using the SYBR Green method with the Bio-Rad CFX96 real-time System (Bio-Rad). Fifteen microliters of reaction volume was used for the real-time PCR assay that consisted of $2 \times(7.5 \mu \mathrm{l})$ Brilliant SYBR Green QPCR Master Mix (Bio-Rad), $2.5 \mu \mathrm{M}$ of primers $(2.5 \mu \mathrm{l}$ each from the stock), and $5 \mu \mathrm{l}$ of template. The thermal conditions consisted of an initial denaturation at $95^{\circ} \mathrm{C}$ for $10 \mathrm{~min}$ followed by 40 cycles of denaturation at $95^{\circ} \mathrm{C}$ for $15 \mathrm{~s}$, annealing at $60^{\circ} \mathrm{C}$ for $30 \mathrm{~s}$, and extension at $72^{\circ} \mathrm{C}$ for $1 \mathrm{~min}$. A melting curve analysis was carried out between 50 and $95^{\circ}$ $\mathrm{C}$ with a plate read every $0.1^{\circ} \mathrm{C}$ after holding the temperature for $5 \mathrm{~s}$. The data were analyzed using Bio-Rad Manager version 2.0, and the results were exported to Microsoft Excel for further analysis. Three endogenous control genes were analyzed for stability in the $\mathrm{CCl}_{4}$ model (Supplementary Information) and $18 \mathrm{~s}$ RNA was chosen as a reliable endogenous control gene. Data normalization relative to the $18 \mathrm{~s}$ RNA subunit was calculated using the comparative $\mathrm{Ct}$
( $\Delta \Delta \mathrm{Ct})$ method to calculate the fold change between the control and experimental groups as previously described. ${ }^{77}$ Primer pairs used for the different mouse and human genes are listed in Table 1.

\section{siRNA Constructs and Cell Transfections}

The following expression plasmids were used: human versican siRNA (Santa Cruz cat. \# sc-41903) and control siRNA (Santa Cruz cat. \# sc-37007). Cells were transfected with specific siRNA as indicated using FugeneHD (Roche cat. \# 04709713001) according to the manufacturer's specification. Transfected cells were analyzed $48 \mathrm{~h}$ post transfection by real-time quantitative PCR (qPCR) or western blot analysis as indicated and described in the Results.

\section{Western Blot Analysis}

Treated cells or liver fragments were washed twice in ice cold phosphate-buffered saline, then lysed and homogenized in RIPA (Boston Bio-products cat. \# BP-115) supplemented with protease inhibitor cocktail tablets (Roche cat. \# 11836153001). Normalized protein samples were mixed with appropriate volume of Laemmli buffer (Boston Bio-product cat. \# BP-110 R), boiled for $5 \mathrm{~min}$, resolved on a $10 \%$ polyacrylamide gel and immuno-blotted with specific antibodies as indicated. Protein bands were analyzed by densitometry, normalized to $\beta$-actin for each lane, using the Kodak Image Station $4000 \mathrm{~mm}$ Pro Chemiluminescence image analyzer and quantitated using ImageJ software (http://imagej.nih.gov/ij/). Molecular weights were estimated in comparison with Precision Plus Protein WesternC Standard (Bio-Rad cat. \# 161-0376). Antibodies used included: $\beta$-actin (Abcam cat. \# ab6276), histone H3 (Abcam cat. \# ab1791), versican (Sigma cat. \# HPA004726), versican DPEAAE neo-epitope antibody (Abcam cat. \# ab19345), ${ }^{55}$ and cleaved poly(ADP-ribose) polymerase antibody (Cell Signaling cat. \# 9544). The Protein Molecular Weight Calculator tool (http://www.sciencegateway.org/tools/pro teinmw.htm) was used to calculate versican core protein fragment sizes based on known amino acid composition.

\section{Lactate Dehydrogenase (LDH) Assay}

The LDH toxicity assay kit (Abcam Cat. \# ab65393) was used according to the manufacturer's specification as recently described. ${ }^{78}$ Briefly, released LDH in culture supernatants of LX2 cells was measured after versican siRNA knockdown. Staurosporine (Cell Signaling cat. \#9953) treatment (10 nM) for $12 \mathrm{~h}$ was used to initiate apoptosis as a positive control for poly(ADP-ribose) polymerase cleavage. The percentage of cytotoxicity was measured by subtracting LDH content in remaining viable cells from total $\mathrm{LDH}$ in untreated controls. The final absorbance was measured at $490 \mathrm{~nm}$. All experiments were performed in triplicate with an equal number of cells $\left(5 \times 10^{6}\right.$ cells $/ 2 \mathrm{ml}$ cell culture medium $)$. 
a
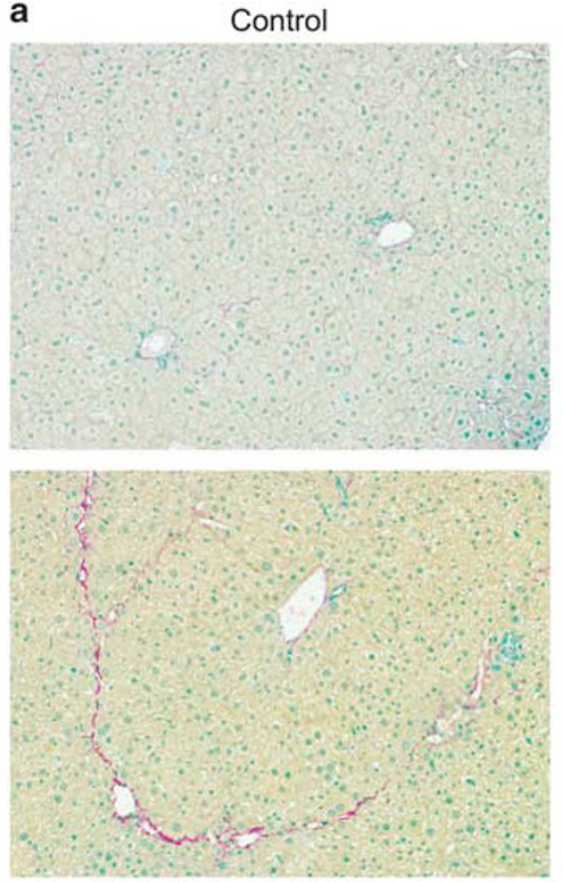

3 day recovery
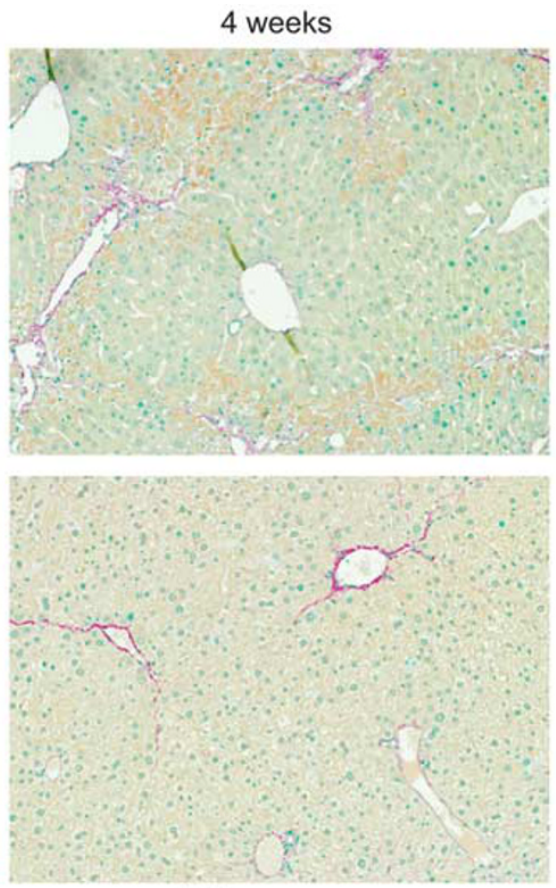

7 day recovery

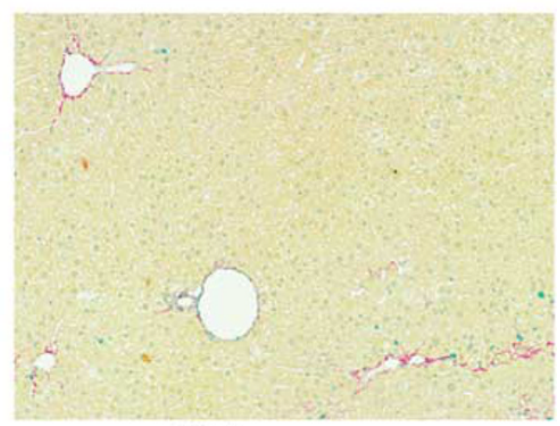

28 day recovery

b

Ishak-Knodell Staging

$\square$ Collagen Quantitation

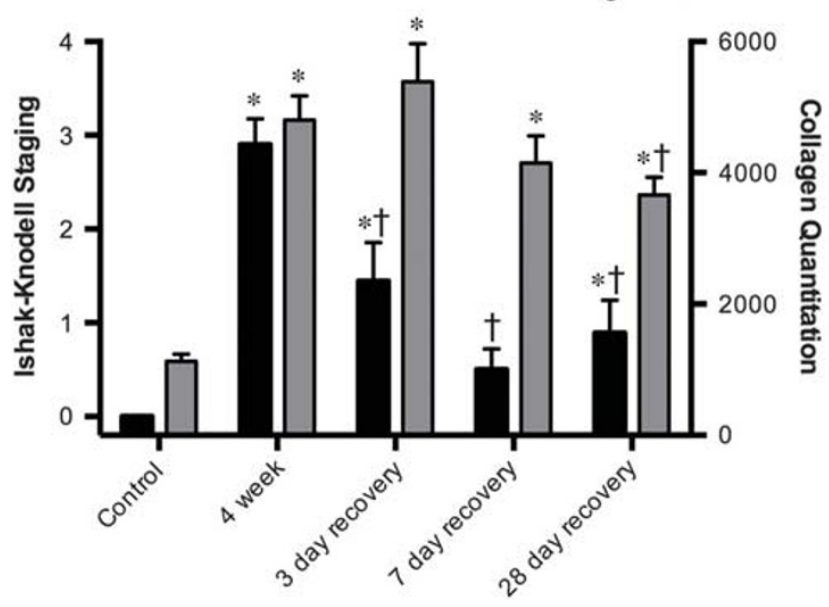

Figure 2 Analysis of collagen deposition and resolution in a reversible model of liver fibrosis. C57BL/6 wild-type mice received i.p. $\mathrm{CCl}_{4}$ or vehicle (olive oil), for 4 weeks treatment, then were allowed to recover for 3, 7, or 28 days, according to the 8-week model of reversible liver fibrogenesis and recovery developed by Iredale et $a^{26}$ ( $n=9-10$ for each group). (a) Histological sections were stained with picrosirius red to visualize collagen. (b) Histological fibrosis staging was performed on sections stained with Masson's trichrome (not shown). One-way analysis of variance was used to determine significant differences for Ishak-Knodell staging or picrosirius red quantitation, compared with control $\left(^{*}\right)$ and compared with 4 weeks of treatment $(\dagger)(P<0.05)$. Mean value per lane is shown and error bars represent the s.e.m.

\section{Statistical Analysis}

All data are expressed as the mean \pm standard error of the mean (s.e.m.). Data were evaluated using two-way (Figure 1) or one-way analysis of variance with Fisher's least significant difference test for multiple comparisons. A value of $P<0.05$ was considered to be statistically significant. Statistical analyses were performed using the GraphPad Prism software program (version 6.0f).

\section{RESULTS}

\section{Versican is Upregulated in Liver Fibrosis and Cirrhosis}

Given the consistent association of versican with many fibroproliferative disorders, we sought to investigate its role in liver fibrosis. We stained normal and cirrhotic human livers for versican and compared these with fibrotic mouse livers after 4 weeks of $\mathrm{CCl}_{4}$ administration. Human and mouse livers both stained faintly in the absence of fibrosis, 

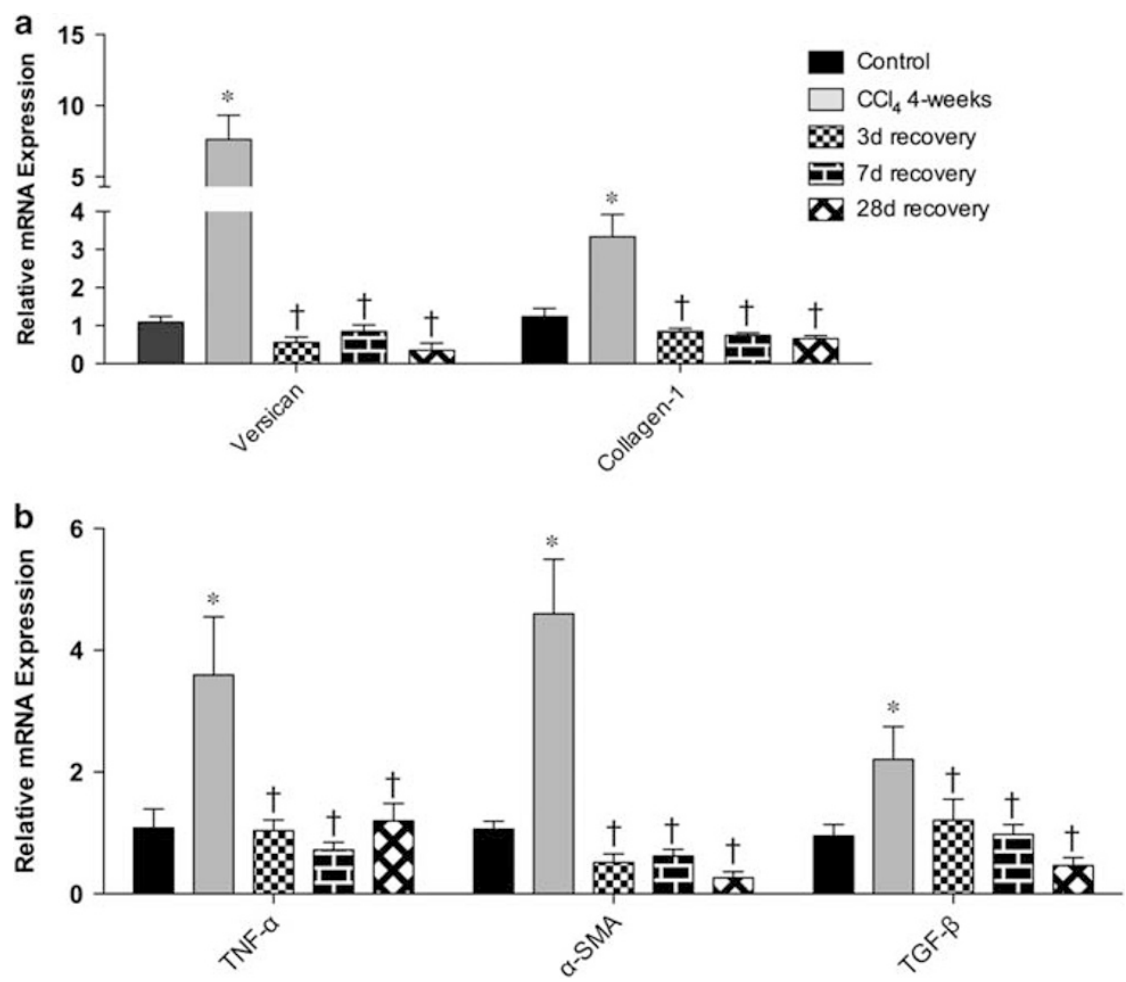

Figure 3 Versican expression increases during liver fibrosis and reduces during recovery. (a) Total RNA was extracted from the livers of control mice, $\mathrm{CCl}_{4}$ 4-week treated mice and mice recovering from $\mathrm{CCl}_{4}$-induced liver injury and analyzed by qPCR for versican and collagen-1 mRNA expression using $18 \mathrm{~s}$ RNA as endogenous control. (b) Liver injury and fibrogenesis were assessed in control mice for TNF- $\alpha$, $a-S M A$, and TGF- $\beta$. Relative mRNA expression and s.e.m. values are shown. Significant differences determined with one-way analysis of variance are indicated compared with control $(*)$ and compared with 4 weeks of treatment $(\dagger)(P<0.05, n=9-10) . C_{C L}$, carbon tetrachloride; $a-S M A$, a-smooth muscle actin; TNF-a, tumor necrosis factor-a; TGF- $\beta$, transforming growth factor- $\beta$.

with a variable staining pattern including both apparent perisinusoidal and hepatocyte cytoplasmic localization. With fibrosis and cirrhosis, both human and mouse livers stained strongly for versican (Figure 1a). This staining was partially subendothelial (perisinusoidal) and stromal, but substantial staining was also cytoplasmic in hepatocytes, particularly in human cirrhotic specimens. Mouse liver stained more ubiquitously for versican under conditions of laboratory induced fibrosis. Quantitation of versican staining confirmed significantly increased versican deposition in both human cirrhosis and the mouse model of liver fibrosis (Figure 1b).

To further investigate the putative role of versican in liver fibrogenesis and recovery, we employed a model of reversible liver fibrosis induced by $\mathrm{CCl}_{4}{ }^{26}$ Histological analysis confirmed the presence of necrosis and inflammation with thick fibrous septae after 4 weeks of injury (Figure 2a). In those animals in whom $\mathrm{CCl}_{4}$ administration was stopped at 4 weeks, there was resolution of inflammation and fibrosis over the following 4 weeks which was statistically significant at 3, 7, and 28 days (Figures $2 \mathrm{a}$ and b). Quantitation of picrosirius red collagen staining indicated that collagen deposition was more sustained and resolution was not as rapid as morphological recovery from injury (Figure $2 b$ ).
After 4 weeks of $\mathrm{CCl}_{4}$-induced injury, liver fibrosis was associated with a significant increase of whole-liver versican mRNA expression (Figure 3a). This was concomitant with an increase in collagen-1, a well described fibrosis gene. The inflammatory response was confirmed by a significant increase in liver TNF- $\alpha$ and fibrogenesis was confirmed by increases in TGF- $\beta$ and $\alpha$-SMA mRNA expression (Figure $3 \mathrm{~b}$ ). During recovery from liver injury, the increase in versican expression decreased to basal levels by day 3 of recovery alongside collagen-1 (Figure 3a). TNF- $\alpha, \alpha$-SMA, and TGF- $\beta$ mRNA decreased to comparable levels with control mice by 3 days after cessation of $\mathrm{CCl}_{4}$ (Figure $3 \mathrm{~b}$ ). Compared with $\alpha$-SMA mRNA expression, there was a more gradual decrease of $\alpha$-SMA protein during recovery (Figure 4a).

\section{Versican Expression and Processing is Dysregulated During Hepatic Fibrosis in Mice}

Given the importance of versican turnover in modulation of cellular functions and fibrosis in other tissues, ${ }^{51,69}$ we next investigated whether versican was being proteolytically processed, using a neo-epitope antibody to the well-documented DPEAAE $\downarrow$ ARRGQ cleavage site (anti-DPEAAE), present in 

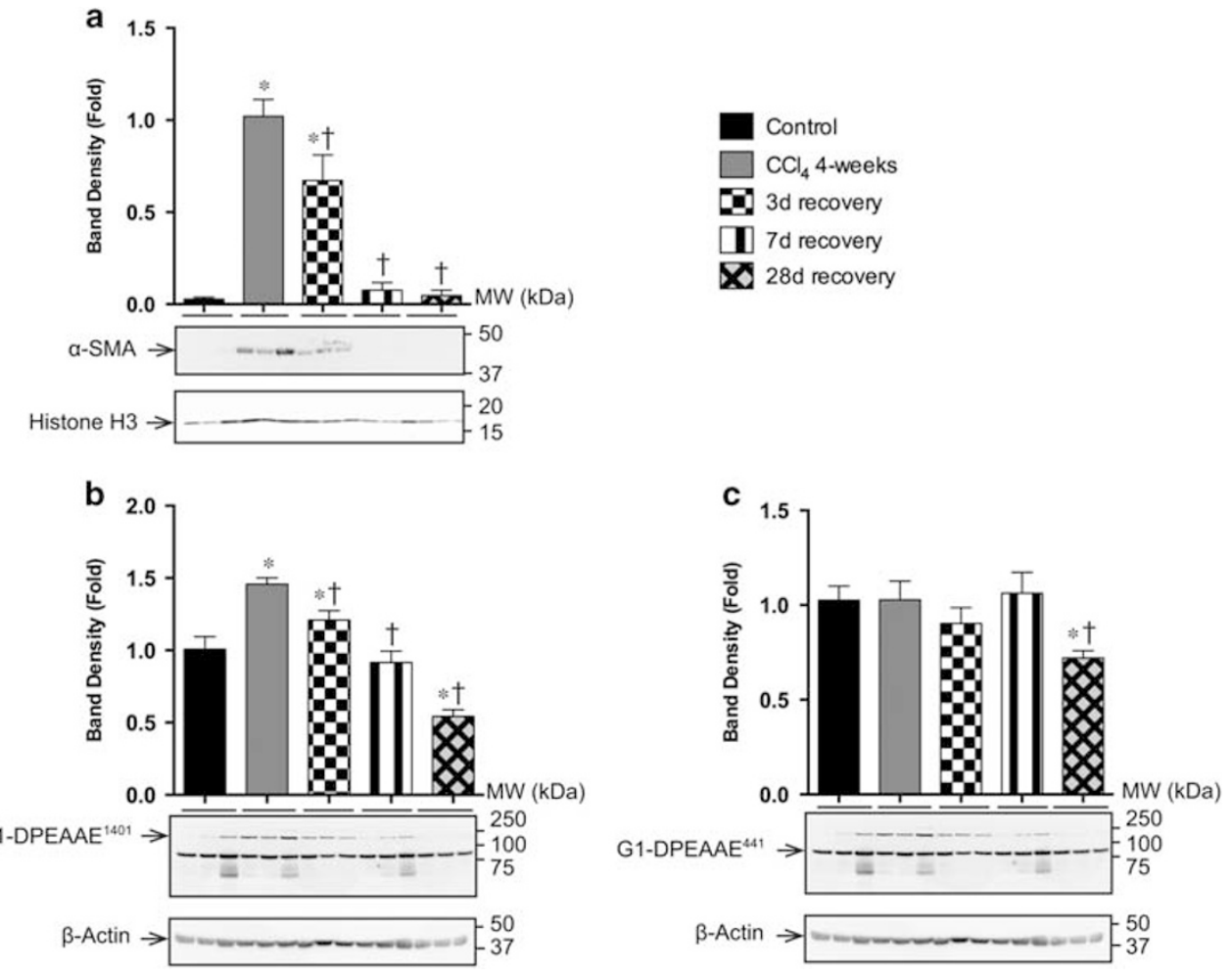

Figure $4 \mathrm{CCl}_{4}$-induced liver fibrosis leads to increase in a-SMA and versican Vo fragment. Total protein was extracted from liver samples of control, $\mathrm{CCl}_{4}$ 4-week treated and $\mathrm{CCl}_{4}$-treated recovering mice. (a) Total protein samples were analyzed for mouse a-SMA expression using histone $\mathrm{H} 3$ as a protein normalizing control. (b and c) Total protein samples were analyzed for the ADAMTS generated versican fragments by western blot analysis using antiDPEAAE neo-epitope antibody and $\beta$-actin as a protein normalizing control. Significant differences determined with one-way analysis of variance are indicated compared with control $\left(^{*}\right)$ and compared with 4 weeks of treatment $(\dagger)(P<0.05)$. Western blots shown demonstrate three representative lanes per time point. Data are representative of 3-4 repeat experiments. Mean band densitometry values per lane are shown ( $n=9-10)$ and error bars represent the s.e.m. ADAMTS, A Disintegrin-like and Metalloproteinase with Thrombospondin-1 motifs; $\mathrm{CCL}_{4}$, carbon tetrachloride; a-SMA, a-smooth muscle actin.

both the V0 and V1 versican isoforms..$^{55}$ We identified that the V0 cleavage product, G1-DPEAAE ${ }^{1401}$, significantly increased at 4 weeks of $\mathrm{CCl}_{4}$, followed by significant decreases during recovery, though it remained elevated compared with control at 3 days of recovery (Figure $4 \mathrm{~b}$ ). Conversely, the V1 cleavage product, G1-DPEAAE ${ }^{41}$ or versikine, showed no significant changes during the induction of fibrosis but a significant decrease at 28 days of recovery (Figure 4c). The V0 and V1 cleavage products were both decreased significantly below control levels at 28 days of recovery (Figures $4 \mathrm{~b}$ and c).

\section{ADAMTS Proteoglycanases are Dysregulated in Liver Fibrosis}

To further investigate putative mechanisms of versican turnover, we next analyzed expression of the ADAMTS proteoglycanses. We found that $\mathrm{CCl}_{4}$ treatment over 4 weeks led to significant increases in liver ADAMTS-5, -9, -15, and -20 mRNA expression and nonsignificant increases in ADAMTS-1, -4 , and -8 (Figures $5 \mathrm{a}-\mathrm{g}$ ). During recovery from liver injury, ADAMTS proteoglycanases exhibited different patterns of regulation. ADAMTS-4, -15 , and -20 decreased rapidly during recovery and at 28 days, ADAMTS -4 and -20 remained decreased compared with 4 weeks of $\mathrm{CCl}_{4}$ treatment (Figures 5b, $\mathrm{f}$ and $\mathrm{g}$ ). In contrast, ADAMTS- 8 was increased compared with 4 weeks of $\mathrm{CCl}_{4}$ treatment at 28 days of recovery and ADAMTS- $5,-8,-9$, and -15 were all increased compared with control at 28 days of recovery (Figures $5 \mathrm{c}-\mathrm{f}$ ).

\section{Versican Expression Increases in Activated Primary Mouse Stellate Cells}

Given that versican was increased significantly during hepatic fibrosis and was reduced during recovery from hepatic injury, we investigated versican expression in HSC activation as they are the main source of ECM in liver injury. Primary HSCs were isolated from 3-week-old C57BL/6 mice followed by activation during tissue culture on plastic. During stellate cell activation, versican expression increased significantly at day 5 compared with day 1 control mouse HSCs (Figure 6a), and appeared to stay elevated through to day 21. Similarly, $\alpha$-SMA and collagen- 1 expression increased significantly at day 5 and appeared to stay elevated through to day 21 (Figures $6 \mathrm{~b}$ and $\mathrm{c}$ ). Throughout the timecourse of HSC activation, ADAMTS- 1 expression changed little with a small 

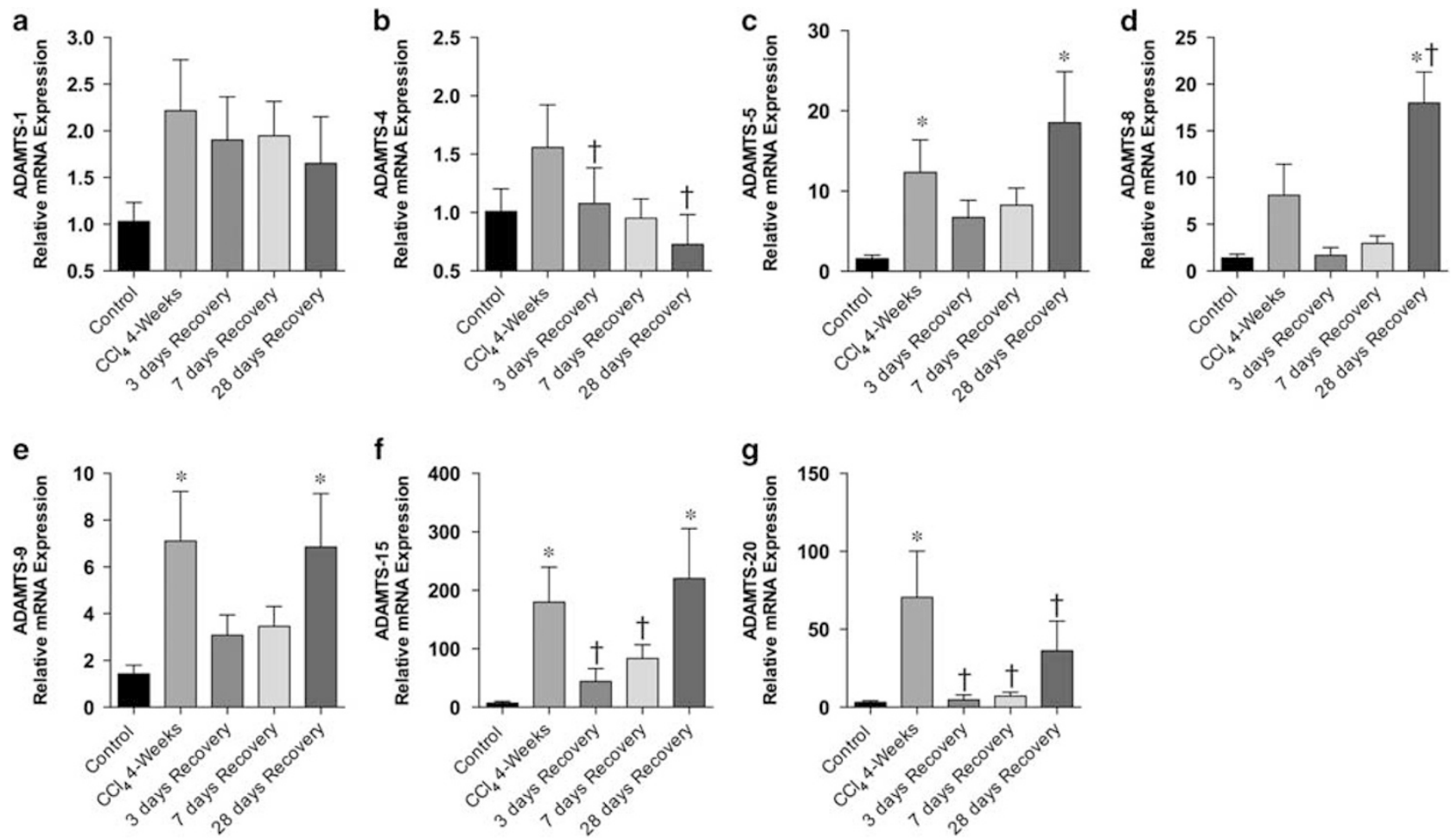

Figure 5 ADAMTS proteoglycanases are dysregulated in $\mathrm{CCl}_{4}$-induced liver fibrosis. Total RNA was extracted from liver samples of control, $\mathrm{CCl}_{4}$ 4-week treated, and $\mathrm{CCl}_{4}$-treated recovering mice. Samples were analyzed by qPCR analysis of mRNA expression for (a) ADAMTS-1, (b) ADAMTS-4, (c) ADAMTS-5, (d) ADAMTS-8, (e) ADAMTS-9, (f) ADAMTS-15, and (g) ADAMTS-20, using $18 \mathrm{~s}$ RNA as endogenous control. Significant differences determined with oneway analysis of variance are indicated compared with control $\left(^{*}\right)$ and compared with 4 weeks of treatment $(\dagger)(P<0.05)$. Data are representative of $3-4$ repeat experiments. Mean value per lane is shown $(n=9-10)$ and error bars represent the s.e.m. ADAMTS, A Disintegrin-like and Metalloproteinase with Thrombospondin-1 motifs; $\mathrm{CCL}_{4}$, carbon tetrachloride.

but significant change at day 14, but ADAMTS-4 expression was significantly downregulated at all time points (Figure 6d). Conversely, TIMP-1 showed significantly elevated expression at day 14 and TIMP-3 was significantly upregulated at days 7 and 21 (Figure 6d) with nonsignificant increased expression of both genes at all other time points. A reduction in ADAMTS-4 along with increased expression of TIMP-1 and -3 should contribute to a net reduction in proteoglycan turnover and a net synthetic environment. Collectively, these data are consistent with the synthesis and deposition of versican and other components of the ECM by activated HSCs.

\section{Transient Knockdown of Versican in HSCs Leads to a Decrease in Profibrotic Gene Expression with no Effect on Cell Viability}

To further characterize the role of versican in liver fibrosis, versican was transiently knocked down in an activated human HSC line (LX2 cells ${ }^{79}$ and in mouse primary HSCs. Versican knockdown in LX2 cells was confirmed by decreased expression of versican mRNA (Supplementary Information). Versican knockdown was associated with a nonsignificant decrease in expression of collagen-1 (Figure 7a), and significant decreases in $\alpha$-SMA (Figure 7b), KI67 (Figure 7c), and TGF- $\beta 1$ (Figure $7 \mathrm{~d}$ ). Versican knockdown led to a reduction in
G1-DPEAAE $^{441}$ (Figure 7e). In contrast to the in vivo model, the versican V0 cleavage product (G1-DPEAAE ${ }^{1428}$ ) was not detected $(n=3)$. Transient knockdown of versican did not stimulate apoptosis as detected by poly(ADP-ribose) polymerase (Figure 7f) or caspase-3 antibody (data not shown) and had no effect on LX2 cell viability (Supplementary Information).

Versican knockdown was also performed with primary mouse HSCs. Similar to LX2 cells, versican knockdown was associated with decreases in the fibrosis-associated genes collagen- 1 and TGF- $\beta 1$ (Figure 7g). Conversely, $\alpha$-SMA expression was not decreased in response to transient versican knockdown in these primary cells (Figure $7 \mathrm{~g}$ ). Collectively these data suggest that versican knockdown inhibits features of fibrogenesis of HSCs cultured on plastic but does not induce apoptosis (in LX2 cells) and does not inhibit activation in primary HSCs (assessed by $\alpha$-SMA expression) under these conditions. These findings are in concert with the hypothesis that versican may modulate HSC cell activation and functions in liver fibrosis.

\section{DISCUSSION}

The pathogenesis of liver fibrosis is closely associated with sustained hepatic inflammation which is evident in human 

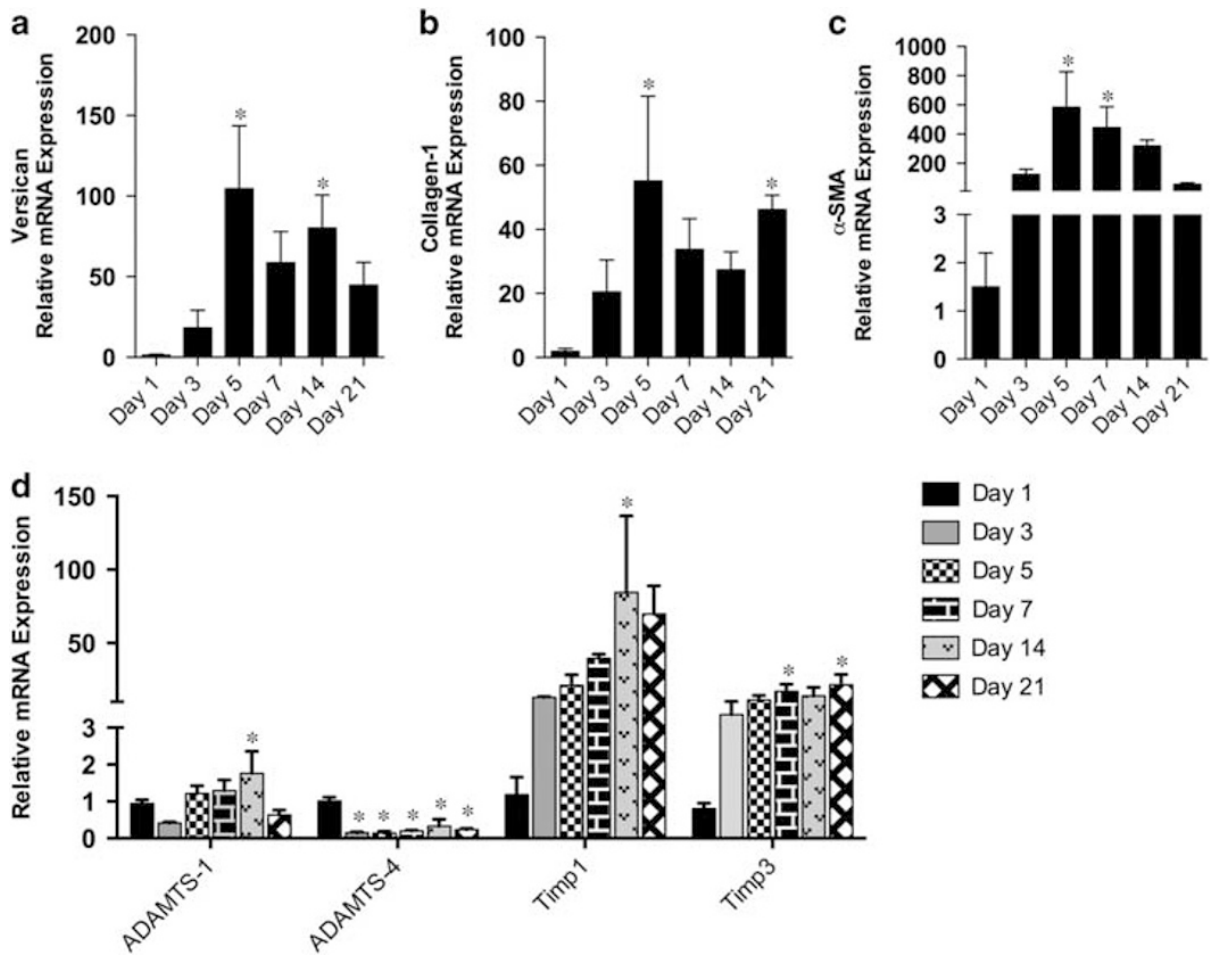

Figure 6 Activation of primary mouse stellate cells leads to increased versican expression. Primary HSCs were isolated from 3- to 6-week-old C57BL/6 mice as indicated in the methods. Isolated primary HSCs were activated by plastic adherence over 1, 3, 5, 7, 14, and 21 days. Total RNA was then extracted from cells and analyzed for (a) versican, (b) collagen-1, and (c) a-SMA mRNA expression using $18 \mathrm{~s}$ RNA as an endogenous control. (d) ADAMTS-1 and - 4 were analyzed alongside TIMP-1 and -3 expression levels, using 18 S RNA as an endogenous control. Significant differences determined with one-way analysis of variance are indicated compared with day $1\left(^{*}\right)(P<0.05)$. Data are representative of 3-4 repeat experiments. Mean value per lane is shown $(n=3)$ and error bars represent the s.e.m. ADAMTS, A Disintegrin-like and Metalloproteinase with Thrombospondin-1 motifs; HSC, hepatic stellate cell; a-SMA, a-smooth muscle actin; TIMP-1, tissue inhibitor of metalloproteinase-1.

disease as well as in experimental liver models. ${ }^{10}$ Many mechanisms leading to liver fibrosis, particularly HSC activation and collagen turnover, have been described. In this report, we present data which suggest for the first time that versican, a large ECM CSPG, is involved in the pathophysiology of hepatic fibrosis and may modulate HSC activation.

We found versican to be dysregulated with significantly increased expression in human cirrhosis and in $\mathrm{CCl}_{4}$-induced fibrosis in mice. Versican upregulation was concomitant with increased expression and dysregulation of the proteoglycanases ADAMTS- $1,-4,-5,-8,-9,-15$, and -20 after 4 weeks of $\mathrm{CCl}_{4}$-induced injury and fibrosis in mice. Following cessation of $\mathrm{CCl}_{4}$ and recovery from injury there was a rapid reduction of versican expression, along with variable changes in ADAMTS expression. Interestingly, a number of the ADAMTS enzymes appeared to increase again at 28 days of recovery, potentially indicating a separate phase of remodeling after the initial recovery period. For ADAMTS-8, this increase was significant compared with both control and 4 weeks of treatment. ADAMTS- 8 is associated with inflammatory remodeling in the vasculature ${ }^{80}$ and displays tumor suppressor activity, ${ }^{81}$ but its role in liver remodeling has not been described.
During recovery from $\mathrm{CCl}_{4}$, there was a rapid reduction of the profibrotic genes $\alpha$-SMA, TGF- $\beta$, and collagen- 1 concomitant with the reduction of versican expression. Whereas the proinflammatory cytokine TNF- $\alpha$ decreased rapidly, ADAMTS-1, an enzyme known to be associated with inflammation and versican processing, ${ }^{82}$ changed minimally. To study versican turnover during the gradual decrease in hepatic inflammation and reversal of fibrosis, we assessed the appearance of versican cleavage products, G1-DPEAAE ${ }^{441}$ (recently dubbed versikine ${ }^{71}$ ) and G1-DPEAAE ${ }^{1401}$, which are generated by the ADAMTS proteoglycanases. We found that the versican V0 fragment was differentially produced during injury and recovery, but the more commonly described V1 fragment (versikine), changed little, with a significant decrease seen only at the 28 days of recovery time point. In this model, it appears that processing of $\mathrm{V} 0$ occurs during injury and gradually decreases over 28 days of recovery, to a level less than control. Given the described roles of versican in inflammation, ${ }^{52-54}$ the prolonged proteolytic turnover of versican is consistent with a period of sustained inflammation during resolution of fibrosis. Additionally, given that engagement of TLR2 by versican appears to be directly involved in the activation and the induction of inflammatory cytokine 

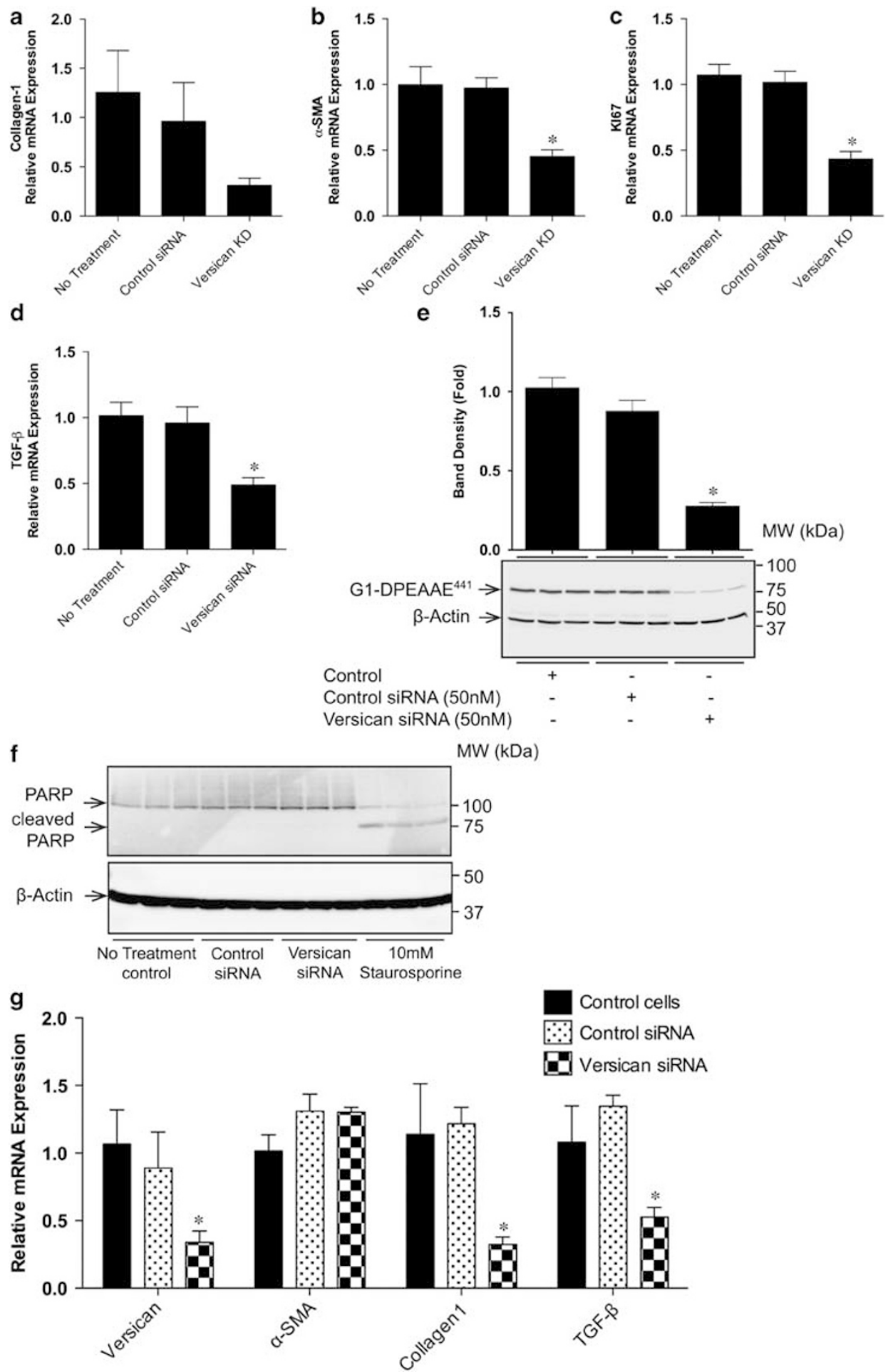

Figure 7 Transient knockdown of versican in LX2 cells leads to a decrease in versican V1 fragment and decreased HSC activation. Total RNA and protein were extracted from LX2 cells $48 \mathrm{~h}$ after transient knockdown of versican or not as indicated. Total RNA was analyzed for (a) collagen-1, (b) a-SMA, (c) KI67, and (d) TGF- $\beta 1$ mRNA expression, respectively, by qPCR using 18 s RNA as an endogenous control. (e) Total protein extracts were analyzed for versican V1 fragment using $\beta$-actin as a protein normalizing control. (f) Total protein was extracted from LX2 cells after versican KD, staurosporine treatment or not as indicated and subjected to western blot analysis for PARP as indicated. (g) Total RNA was extracted from primary mouse HSC cultures $48 \mathrm{~h}$ after knockdown of versican or not (control) as indicated. mRNA expression was analyzed by qPCR for versican, $\alpha-S M A$, collagen-1, and TGF- $\beta 1$ using $18 \mathrm{~s}$ RNA as an endogenous control. Significant differences determined with one-way analysis of variance are indicated compared with no treatment control $\left(^{*}\right)(P<0.05)$. Data are representative of 3-4 repeat experiments. The mean band densitometry value per lane is shown $(n=3)$ in $(\mathbf{e})$ and error bars represent the s.e.m. HSC, hepatic stellate cell; $\alpha$-SMA, $\alpha$-smooth muscle actin; TGF- $\beta$, transforming growth factor- $\beta$; PARP, poly(ADP-ribose)polymerase. 
secretion, ${ }^{83}$ our observation that hepatic fibrosis leads to a significant increase in versican fragments may indicate that versican processing contributes to the inflammatory response.

The prolonged proteolytic turnover of versican is associated with sustained expression of a number of the proteoglycan-degrading ADAMTS enzymes, most increasing during injury and several (ADAMTS-5, -8, -9, and -15) increasing a second time at the later recovery time point of 28 days. This second increase in proteoglycanases corresponds with the later decreases in levels of versican cleavage products below control levels. The physiological significance of these changes is not yet clear. However, we also found that there was a difference between the morphological recovery from $\mathrm{CCl}_{4}$ and the loss of collagen staining, the latter being a more prolonged process. $\alpha$-SMA protein was also not lost as rapidly as gene expression was downregulated. Thus it is possible that protein changes in the ECM endure beyond morphological recovery and might predispose to future injury. It is currently unclear if the proteolytic fragments of versican are bioactive in this model as has been shown in other tissues, but they may play a role in late remodeling of the ECM during resolution or maintenance of hepatic fibrosis. Collectively, these data shed further light on the mechanisms of ECM remodeling and their importance during the resolution of hepatic fibrosis.

In our experiments we consistently found versican G1-DPEAAE ${ }^{441}$ or versikine, to run at an apparent molecular weight of $\sim 80 \mathrm{kDa}$. In previous literature this fragment has been referred to as a $70 \mathrm{kDa}$ fragment. Based on core protein primary sequence, masses for the human and mouse G1-DPEAAE ${ }^{441} \mathrm{~V} 1$ fragments are calculated to be 48.95 and $49.06 \mathrm{kDa}$, respectively. For the larger V0 fragments previously reported as an $\sim 220 \mathrm{kDa}$ fragment, human G1-DPEAAE $^{1428}$ is calculated to be $156.73 \mathrm{kDa}$ and mouse G1-DPEAAE $^{1401}$ is calculated to be $153.26 \mathrm{kDa}$. The differences in mobility may be due to the presence of $\mathrm{N}$ - and O-linked glycosylations, or variations in its electrophoretic mobility due to the extremely acidic pI of the core protein. ${ }^{36,41}$

To further characterize the role of versican in hepatic fibrogenesis, we isolated and activated primary mouse HSCs by plastic adherence. We found that following primary HSC activation, there was a significant upregulation of versican alongside other genes, $\alpha$-SMA and collagen- 1 , known to be upregulated in hepatic fibrosis. The expression of the versican cleaving ADAMTS-4 enzyme was significantly downregulated during activation on plastic and TIMP-1 and -3 were significantly upregulated, indicating a net synthetic environment. Out of the family of four TIMPs, TIMP-3 is known to specifically inhibit the ADAMTS enzymes ${ }^{84,85}$ and has previously been associated with TNF activity, sustained liver inflammation, and impaired liver regeneration. ${ }^{86}$ Our data support roles for TIMP-1 and -3 in inhibiting ADAMTS and MMP proteases during HSC activation (already established for MMPs), fibrogenesis, and stimulation of inflammation.
To assess the impact of therapeutically targeting versican in the treatment of hepatic fibrosis, we transiently knocked down versican in an activated human HSC line (LX2 cells) and primary mouse HSCs. Transient knockdown of versican in LX2 cells was associated with a significant decrease in versican mRNA and versikine fragments with no significant effect on cell viability. Additionally, transient knockdown of versican was associated with a decrease in proliferation of activated cells and markers of activation: collagen-1, $\alpha$-SMA, KI67, and TGF- $\beta$. In primary mouse HSCs, transient knockdown of versican had similar effects on cell activation; however, it did not reduce $\alpha$-SMA expression. It may be that the efficiency and/or duration of transient knockdown was insufficient to induce inhibition of phenotypic changes in the activating primary HSCs, whereas in the already activated HSC line, transient knockdown of versican was sufficient to inhibit markers of activation as well as markers of fibrogenesis. These observations indicate that versican has bioactivity in HSCs and loss of versican inhibits expression of fibrogenic genes and may inhibit HSC activation.

In summary, we present data indicating that during liver fibrosis, versican increases significantly and may be important in modulating HSC activation, hepatic inflammation, and fibrosis. Taken together, our findings highlight versican expression and processing as new dimensions in our understanding of the processes of hepatic inflammation and fibrosis. Future work will need to investigate whether this proteoglycan is similarly implicated in other models of chronic liver injury such as alcohol and diet-induced liver fibrosis. Therapeutic interventions aimed at modulating versican expression or turnover could be useful in ameliorating liver fibrosis. The development of future antifibrotic treatments for liver disease needs to take into account the role and proteolytic turnover of versican and other proteoglycans. A deeper understanding of the expression and turnover of versican and other proteoglycans may also lead to the development of new non-invasive markers of liver fibrosis.

Supplementary Information accompanies the paper on the Laboratory Investigation website (http://www.laboratoryinvestigation.org)

\section{ACKNOWLEDGMENTS}

We thank Sarah Gray and R Luke Harris for insightful comments and suggestions throughout this study. We are grateful to Scott $L$ Friedman for kindly providing the human hepatic stellate cell line (LX2 cells). We thank Lydia Troc and staff of the animal facility at the University of Northern British Columbia for care of mice used in this study. This work was supported by startup funds to PJW from the Northern Medical Program (http://www. unbc.ca/northern-medical-program).

\section{DISCLOSURE/CONFLICT OF INTEREST}

The authors declare no conflict of interest.

1. Zakhari S. Bermuda Triangle for the liver: alcohol, obesity, and viral hepatitis. J Gastroenterol Hepatol 2013;28:18-25. 
2. Friedman SL. Preface. Hepatic fibrosis: pathogenesis, diagnosis, and emerging therapies. Clin Liver Dis. 2008;12:xiii-xiv.

3. Liberal R, Grant CR, Mieli-Vergani $G$ et al. Autoimmune hepatitis: a comprehensive review. J Autoimmun 2013;41:126-139.

4. Szabo G, Aloman C, Polyak SJ et al. Hepatitis C infection and alcohol use: a dangerous mix for the liver and antiviral immunity. Alcohol Clin Exp Res 2006;30:709-719.

5. Lee UE, Friedman SL. Mechanisms of hepatic fibrogenesis. Best Pract Res Clin Gastroenterol 2011;25:195-206.

6. Iredale JP. Models of liver fibrosis: exploring the dynamic nature of inflammation and repair in a solid organ. J Clin Invest 2007;117: 539-548.

7. Hernandez-Gea V, Friedman SL. Pathogenesis of liver fibrosis. Annu Rev Pathol 2011;6:425-456.

8. Gao B, Seki E, Brenner DA et al. Innate immunity in alcoholic liver disease. Am J Physiol Gastrointest Liver Physiol 2011;300:G516-G525.

9. Kowalski-Saunders $\mathrm{P}$, Winwood $\mathrm{P}$, Arthur $\mathrm{M}$ et al. Reversible inhibition of albumin production by rat hepatocytes maintained on a lamininrich gel (Engelbreth-Holm-Swarm) in response to secretory products of Kupffer cells and cytokines. Hepatology 1992;16:733-741.

10. Pellicoro A, Ramachandran P, Iredale JP et al. Liver fibrosis and repair: immune regulation of wound healing in a solid organ. Nat Rev Immunol 2014:14:181-194.

11. Friedman SL. Hepatic fibrosis-overview. Toxicology 2008;254: 120-129.

12. Gaça MDA, Zhou $X$, Issa $R$ et al. Basement membrane-like matrix inhibits proliferation and collagen synthesis by activated rat hepatic stellate cells: evidence for matrix-dependent deactivation of stellate cells. Matrix Biol 2003;22:229-239.

13. Zhou $X$, Murphy FR, Gehdu $N$ et al. Engagement of $\operatorname{av} \beta 3$ integrin regulates proliferation and apoptosis of hepatic stellate Cells. J Biol Chem 2004;279:23996-24006.

14. Olaso E, Ikeda K, Eng FJ et al. DDR2 receptor promotes MMP-2mediated proliferation and invasion by hepatic stellate cells. J Clin Invest 2001;108:1369-1378.

15. Friedman SL, Roll FJ, Boyles J et al. Maintenance of differentiated phenotype of cultured rat hepatic lipocytes by basement membrane matrix. J Biol Chem 1989;264:10756-10762.

16. Olsen AL, Bloomer SA, Chan EP et al. Hepatic stellate cells require a stiff environment for myofibroblastic differentiation. Am J Physiol Gastrointest Liver Physiol 2011;301:G110-G118.

17. Wells $R$. The role of matrix stiffness in hepatic stellate cell activation and liver fibrosis. J Clin Gastroenterol 2005:39:S158-S161.

18. Serpaggi J, Carnot F, Nalpas B et al. Direct and indirect evidence for the reversibility of cirrhosis. Hum Pathol 2006;37:1519-1526.

19. Hammel P, Couvelard A, O'Toole D et al. Regression of liver fibrosis after biliary drainage in patients with chronic pancreatitis and stenosis of the common bile duct. N Engl J Med 2001;344: 418-423.

20. Dufour J-F, DeLellis R, Kaplan MM. Reversibility of hepatic fibrosis in autoimmune hepatitis. Ann Intern Med 1997;127:981-985.

21. Muretto $P$, Angelucci E, Lucarelli G. Reversibility of cirrhosis in patients cured of thalassemia by bone marrow transplantation. Ann Intern Med 2002;136:667-672.

22. McCrudden R, Iredale JP. Liver fibrosis, the hepatic stellate cell and tissue inhibitors of metalloproteinases. Histol Histopathol 2000;15: 1159-1168.

23. Friedman SL. Molecular regulation of hepatic fibrosis, an integrated cellular response to tissue injury. J Biol Chem 2000;275:2247-2250.

24. Elsharkawy AM, Oakley F, Mann DA. The role and regulation of hepatic stellate cell apoptosis in reversal of liver fibrosis. Apoptosis 2005;10: 927-939.

25. Iredale $\mathrm{J}$, Benyon $\mathrm{R}$, Arthur $\mathrm{M}$ et al. Tissue inhibitor of metalloproteinase-1 messenger RNA expression is enhanced relative to interstitial collagenase messenger RNA in experimental liver injury and fibrosis. Hepatology 1996;24:176-184.

26. Iredale JP, Benyon RC, Pickering J et al. Mechanisms of spontaneous resolution of rat liver fibrosis. Hepatic stellate cell apoptosis and reduced hepatic expression of metalloproteinase inhibitors. J Clin Invest 1998:102:538-549.

27. Yoshiji H, Kuriyama S, Miyamoto $\mathrm{Y}$ et al. Tissue inhibitor of metalloproteinases-1 promotes liver fibrosis development in a transgenic mouse model. Hepatology 2000;32:1248-1254.
28. Yoshiji H, Kuriyama $\mathrm{S}$, Yoshii $\mathrm{J}$ et al. Tissue inhibitor of metalloproteinases-1 attenuates spontaneous liver fibrosis resolution in the transgenic mouse. Hepatology 2002;36:850-860.

29. Siller-López F, Sandoval A, Salgado $S$ et al. Treatment with human metalloproteinase-8 gene delivery ameliorates experimental rat liver cirrhosis. Gastroenterology 2004;126:1122-1133.

30. Iozzo RV. Matrix proteoglycans: from molecular design to cellular function. Annu Rev Biochem 1998;67:609-652.

31. Gressner AM. Activation of proteoglycan synthesis in injured liver-a brief review of molecular and cellular aspects. Eur J Clin Chem Clin Biochem 1994;32:225-237.

32. Gressner AM, Weiskirchen R. Modern pathogenetic concepts of liver fibrosis suggest stellate cells and TGF- $\beta$ as major players and therapeutic targets. J Cell Mol Med 2006;10:76-99.

33. Bissell DM. Primary hepatocyte culture: substratum requirements and production of matrix components. Fed Proc 1981;40:2469-2473.

34. Krishnan A, Li X, Kao W-Y et al. Lumican, an extracellular matrix proteoglycan, is a novel requisite for hepatic fibrosis. Lab Invest 2012:92:1712-1725.

35. Meyer D, Krull N, Dreher K et al. Biglycan and decorin gene expression in normal and fibrotic rat liver: cellular localization and regulatory factors. Hepatology 1992;16:204-216.

36. Dours-Zimmermann MT, Zimmermann DR. A novel glycosaminoglycan attachment domain identified in two alternative splice variants of human versican. J Biol Chem 1994;269:32992-32998.

37. Bode-Lesniewska B, Dours-Zimmermann $M$, Odermatt $B$ et al. Distribution of the large aggregating proteoglycan versican in adult human tissues. J Histochem Cytochem 1996;44:303-312.

38. Szende B, Lapis K, Kovalszky I et al. Role of the modified (glycosaminoglycan producing) perisinusoidal fibroblasts in the CCl4induced fibrosis of the rat liver. In Vivo 1992;6:355-361.

39. Younossi ZM, Gorreta F, Ong JP et al. Hepatic gene expression in patients with obesity-related non-alcoholic steatohepatitis. Liver Int 2005:25:760-771.

40. Maurice SB, Crick CL, Kim W-C et al. Versican expression and turnover in hepatic stellate cell activation. Can J Gastroenterol 2012;26:108A.

41. Zimmermann DR, Ruoslahti E. Multiple domains of the large fibroblast proteoglycan, versican. EMBO J 1989;8:2975-2981.

42. Mjaatvedt $\mathrm{CH}$, Yamamura $\mathrm{H}$, Capehart $\mathrm{AA}$ et al. The Cspg2 gene, disrupted in the hdf mutant, is required for right cardiac chamber and endocardial cushion formation. Dev Biol 1998;202:56-66.

43. Choocheep K, Hatano S, Takagi H et al. Versican facilitates chondrocyte dfifferentiation and regulates joint morphogenesis. J Biol Chem 2010;285:21114-21125.

44. Roberts CR. Versican in the cell biology of pulmonary fibrosis. In: Garg HG, Roughley PJ, Hales CA (eds). Proteoglycans in Lung Disease, Vol. 168. Marcel Dekker: New York, 2003, pp 191-212.

45. Bensadoun E, Burke A, Hogg J et al. Proteoglycan deposition in pulmonary fibrosis. Am J Respir Crit Care Med 1996;154:1819-1828.

46. Bensadoun $\mathrm{E}$, Burke $\mathrm{A}$, Hogg J et al. Proteoglycans in granulomatous lung diseases. Eur Respir J 1997;10:2731-2737.

47. Wight TN, Merrilees MJ. Proteoglycans in atherosclerosis and restenosis: key roles for versican. Circ Res 2004;94:1158-1167.

48. Ricciardelli C, Sakko AJ, Ween MP et al. The biological role and regulation of versican levels in cancer. Cancer Metastasis Rev 2009;28: 233-245.

49. Maurice SB. Metalloproteinase Cleavage of Versican at the Fibroblast Cell Surface. University of British Columbia: Vancouver, 2009.

50. Lin H-M, Chatterjee A, Lin Y-H et al. Genome wide expression profiling identifies genes associated with colorectal liver metastasis. Oncol Rep 2007:17:1541-1549.

51. Wight TN, Kinsella MG, Evanko SP et al. Versican and the regulation of cell phenotype in disease. Biochim Biophys Acta 2014;1840: 2441-2451.

52. Zhang Z, Miao L, Wang L. Inflammation amplification by versican: the first mediator. Int J Mol Sci 2012;13:6873-6882.

53. Wight TN, Kang I, Merrilees MJ. Versican and the control of inflammation. Matrix Biol 2014;35:152-161.

54. Kim S, Takahashi H, Lin W-W et al. Carcinoma-produced factors activate myeloid cells through TLR2 to stimulate metastasis. Nature 2009;457: 102-106.

55. Sandy JD, Westling J, Kenagy RD et al. Versican V1 proteolysis in human aorta in vivo occurs at the Glu441-Ala442 bond, a site that is 
cleaved by recombinant ADAMTS-1 and ADAMTS-4. J Biol Chem 2001;276:13372-13378.

56. Somerville RPT, Longpre J-M, Jungers KA et al. Characterization of ADAMTS-9 and ADAMTS-20 as a distinct ADAMTS subfamily related to Caenorhabditis elegans GON-1. J Biol Chem 2003;278:9503-9513.

57. Longpré J-M, McCulloch DR, Koo B-H et al. Characterization of proADAMTS5 processing by proprotein convertases. Int J Biochem Cell Biol 2009:41:1116-1126.

58. Dancevic CM, Fraser FW, Smith $A D$ et al. Biosynthesis and expression of a Disintegrin-like and Metalloproteinase Domain with Thrombospondin-1 Repeats-15: a novel versican-cleaving proteoglycanase. J Biol Chem 2013;288:37267-37276.

59. Apte SS. A disintegrin-like and metalloprotease (reprolysin type) with thrombospondin type 1 motifs: the ADAMTS family. Int J Biochem Cell Biol 2004;36:981-985.

60. Westling J, Gottschall PE, Thompson VP et al. ADAMTS4 (aggrecanase1) cleaves human brain versican $V 2$ at Glu405-Gln406 to generate glial hyaluronate binding protein. Biochem J 2004;377:787-795.

61. Jönsson-Rylander A-C, Nilsson T, Fritsche-Danielson $R$ et al. Role of ADAMTS-1 in atherosclerosis: remodeling of carotid artery, immunohistochemistry, and proteolysis of versican. Arterioscler Thromb Vasc Biol 2005;25:180-185.

62. Ito $\mathrm{K}$, Shinomura $\mathrm{T}$, Zako $\mathrm{M}$ et al. Multiple forms of mouse PG-M, a large chondroitin sulfate proteoglycan generated by alternative splicing. J Biol Chem 1995;270:958-965.

63. McCulloch DR, Nelson CM, Dixon $\sqcup$ et al. ADAMTS metalloproteases generate active versican fragments that regulate interdigital web regression. Dev Cell 2009;17:687-698.

64. Dupuis LE, McCulloch DR, McGarity JD et al. Altered versican cleavage in ADAMTS5 deficient mice; a novel etiology of myxomatous valve disease. Dev Biol 2011;357:152-164.

65. Kern CB, Norris RA, Thompson RP et al. Versican proteolysis mediates myocardial regression during outflow tract development. Dev Dyn 2007;236:671-683.

66. Kern CB, Twal WO, Mjaatvedt $\mathrm{CH}$ et al. Proteolytic cleavage of versican during cardiac cushion morphogenesis. Dev Dyn 2006;235: 2238-2247.

67. Enomoto $\mathrm{H}$, Nelson CM, Somerville RPT et al. Cooperation of two ADAMTS metalloproteases in closure of the mouse palate identifies a requirement for versican proteolysis in regulating palatal mesenchyme proliferation. Development 2010;137:4029-4038.

68. Capehart AA. Proteolytic cleavage of versican during limb joint development. Anat Rec 2010;293:208-214.

69. Frey $\mathrm{H}$, Schroeder $\mathrm{N}$, Manon-Jensen $\mathrm{T}$ et al. Biological interplay between proteoglycans and their innate immune receptors in inflammation. FEBS J 2013;280:2165-2179.

70. Maquart F-X, Pasco S, Ramont $\mathrm{L}$ et al. An introduction to matrikines: extracellular matrix-derived peptides which regulate cell activity: implication in tumor invasion. Crit Rev Oncol Hematol 2004;49:199-202.
71. Nandadasa S, Foulcer S, Apte SS. The multiple, complex roles of versican and its proteolytic turnover by ADAMTS proteases during embryogenesis. Matrix Biol 2014;35:34-41.

72. Perides G, Asher RA, Lark MW et al. Glial hyaluronate-binding protein: a product of metalloproteinase digestion of versican? Biochem J 1995;312:377-384

73. Kawser CA, Iredale JP, Winwood PJ et al. Rat hepatic stellate cell expression of alpha2-macroglobulin is a feature of cellular activation: implications for matrix remodelling in hepatic fibrosis. Clin Sci 1998;95: 179-186.

74. Friedman SL, Roll FJ. Isolation and culture of hepatic lipocytes, Kupffer cells, and sinusoidal endothelial cells by density gradient centrifugation with Stractan. Anal Biochem 1987;161:207-218.

75. Friedman SL, Roll FJ, Boyles J et al. Hepatic lipocytes: the principal collagen-producing cells of normal rat liver. Proc Natl Acad Sci USA 1985;82:8681-8685.

76. Weiskirchen R, Gressner AM. Isolation and culture of hepatic stellate cells. Methods Mol Med 2005;117:99-113.

77. Livak KJ, Schmittgen TD. Analysis of relative gene expression data using real-time quantitative PCR and the 2(-Delta Delta $C(T))$ Method. Methods 2001;25:402-408.

78. Bukong TN, Hou W, Kodys $K$ et al. Ethanol facilitates hepatitis $C$ virus replication via up-regulation of GW182 and heat shock protein 90 in human hepatoma cells. Hepatology 2013;57:70-80.

79. Xu L, Hui AY, Albanis E et al. Human hepatic stellate cell lines, LX-1 and LX-2: new tools for analysis of hepatic fibrosis. Gut 2005;54:142-151.

80. Wågsäter $\mathrm{D}$, Björk $\mathrm{H}$, Zhu $\mathrm{C}$ et al. ADAMTS-4 and -8 are inflammatory regulated enzymes expressed in macrophage-rich areas of human atherosclerotic plaques. Atherosclerosis 2008;196:514-522.

81. Choi GCG, Li J, Wang Y et al. The metalloprotease ADAMTS8 displays antitumor properties through antagonizing EGFR-MEK-ERK signaling and is silenced in carcinomas by $\mathrm{CpG}$ methylation. Mol Cancer Res 2014;12:228-238.

82. Kuno K, Kanada N, Nakashima E et al. Molecular cloning of a gene encoding a new type of metalloproteinase-disintegrin family protein with thrombospondin motifs as an inflammation associated gene. J Biol Chem 1997;272:556-562.

83. Wang $W, X u$ G-L, Jia W-D et al. Ligation of TLR2 by versican: a link between inflammation and metastasis. Arch Med Res 2009;40: 321-323.

84. $\mathrm{Yu}$ W-H, $\mathrm{Yu}$ S-sC, Meng $\mathrm{Q}$ et al. TIMP-3 binds to sulfated glycosaminoglycans of the extracellular matrix. J Biol Chem 2000;275: 31226-31232.

85. Kashiwagi $\mathrm{M}$, Tortorella $\mathrm{M}$, Nagase $\mathrm{H}$ et al. TIMP-3 is a potent inhibitor of Aggrecanase 1 (ADAM-TS4) and Aggrecanase 2 (ADAM-TS5). J Biol Chem 2001:276:12501-12504.

86. Mohammed FF, Smookler DS, SEM Taylor et al. Abnormal TNF activity in Timp3-/- mice leads to chronic hepatic inflammation and failure of liver regeneration. Nat Genet 2004;36:969-977. 